\title{
An inverse relationship between plasma glutathione concentration and fasting glycemia in patients with coronary artery disease and concomitant type 2 diabetes: A pilot study
}

\author{
Kamil Karolczak ${ }^{1, A-F}$, Paweł Kubalczyk 2, B, E, F, Rafał Głowacki2, B, E, F, Robert Pietruszyński3, B, E, F, Cezary Watała ${ }^{1, B-F}$ \\ ${ }^{1}$ Department of Hemostatic Disorders, Medical University of Lodz, Poland \\ ${ }^{2}$ Department of Environmental Chemistry, Faculty of Chemistry, University of Lodz, Poland \\ ${ }^{3}$ Department of Radiological and Nuclear Diagnostics and Therapy, Central Veterans' Hospital, Łódź, Poland \\ A - research concept and design; B - collection and/or assembly of data; C - data analysis and interpretation; \\ $D$ - writing the article; $E$ - critical revision of the article; $F$ - final approval of the article
}

Address for correspondence

Kamil Karolczak

E-mail:kamilkarolczak@gmail.com

\section{Funding sources}

The research was sponsored by grant

No. 502-03/6-020-01/502-64-058

from the Medical University of Lodz (Poland)

and the Polish Society of Metabolic Diseases,

and partially by funds from the National Center

of Science (Kraków, Poland; UM0-2012/07/N/

NZ1/03140).

Conflict of interest

None declared

Received on February 12, 2016

Reviewed on September 22, 2016

Accepted on September 29, 2016

\begin{abstract}
Background. There have been occasional reports indicating that plasma concentrations of reduced glutathione (GSH) may be associated in some way with blood glucose. This relationship, however, has not hitherto been explored in the blood plasma of patients with coronary artery disease (CAD).

Objectives. The aim of this study was to evaluate potential associations of fasting glycemia and peripheral blood plasma GSH concentrations in CAD-free and CAD-affected subjects.

Material and methods. In blood samples obtained from patients with CAD, defined by coronary angiography and/or echocardiography, and from an age-matched control group of patients with a confirmation of no coronary artery occlusion and with no history of cardiovascular events, plasma concentrations of glucose and reduced glutathione were analyzed by routine laboratory diagnostic methods and high performance liquid chromatography (HPLC), respectively.

Results. The results showed that in the CAD patients, but not in the non-CAD controls, fasting glycemia is negatively associated with plasma levels of GSH $(r=-0.328 ; p=0.011)$. Moreover, in the CAD-affected subjects (but not in the controls) the presence of type 2 diabetes mellitus significantly discriminated plasma levels of GSH ( $r_{p}=-0.125 ; p=0.350$, between GSH and glucose adjusted for the occurrence of diabetes).

Conclusions. The study suggests that GSH may be an important factor contributing to glucose metabolism in CAD patients. Hence, it may be considered a significant therapeutic target in strategies aimed at improving glycemic control in CAD-affected subjects.
\end{abstract}

Key words: atherosclerosis, glucose, glutathione, cardiovascular risk factors, diabetes mellitus

DOI

10.17219/acem/65441

Copyright

Copyright by Author(s)

This is an article distributed under the terms of the

Creative Commons Attribution Non-Commercial License

(http://creativecommons.org/licenses/by-nc-nd/4.0/) 


\section{Introduction}

Impaired fasting glycemia is associated with the severity of coronary artery disease (CAD). ${ }^{1,2}$ Maintaining proper proportions between glucose utilization in essential cell metabolism and glucose supply in the daily diet is one of the key factors protecting against the development of CAD, as well as minimizing the risk of CAD aggravation in the cases of existing CAD. The prime example of the association between glucose metabolism and CAD is diabetes mellitus (DM), which is considered one of the main CAD risk factors. ${ }^{2-4}$

One of the leading hypotheses elucidating the relationship between disturbances in glucose metabolism and CAD posits that oxidative stress is a link between hyperglycemia and CAD. This association, which is observed in subjects with disturbed glucose metabolism, is also seen in the general population. ${ }^{5,6}$ Despite huge progress in understanding hyperglycemia-induced cardiovascular pathogenesis, the exact biochemical pathway(s) linking glucose with oxidative stress in CAD are still not completely understood. ${ }^{7}$ Particular attention in this regard can be paid to glutathione (GSH), one of the low-molecular-weight thiols, formed by 3 amino acids (tripeptide). GSH constitutes the main "ingredient" of both extracellular redox buffers (including the blood plasma) and intracellular ones. Its importance in the pathogenesis, progress and treatment of CAD has been shown in a few recent works. ${ }^{8-10}$ Some reports clearly suggest a relationship between glycemia and GSH. ${ }^{7,11}$ Nevertheless, the possible association of plasma GSH levels and glucose concentration has not yet been evaluated in CAD patients with and without concomitant type 2 diabetes mellitus. The aim of the present study was to evaluate this relationship.

\section{Material and methods}

\section{Chemicals}

Sodium hydroxide $(\mathrm{NaOH})$, hydrochloric acid $(\mathrm{HCl})$, sodium hydrogen phosphate heptahydrate $\left(\mathrm{Na}_{2} \mathrm{HPO}_{4} \cdot 7 \mathrm{H}_{2} \mathrm{O}\right)$, sodium dihydrogen phosphate dihydrate $\left(\mathrm{NaH}_{2} \mathrm{PO}_{4} \cdot 2 \mathrm{H}_{2} \mathrm{O}\right)$ and HPLC-grade acetonitrile were obtained from J.T. Baker Chemicals (Deventer, the Netherlands). Trichloroacetic acid (TCA), perchloric acid (PCA) and tris-(2-carboxyethyl)phosphine (TCEP) were obtained from the Merck Group (Darmstadt, Germany). Sodium dodecyl sulfate (SDS), Ellman's reagent 5,5'-dithiobis-(2-nitrobenzoic acid) (DTNB), glutathione (reduced), 2,4-dinitrophenylhydrazine (Brady's reagent, DNPH), ethanol, ethyl acetate and guanidine hydrochloride were from Sigma-Aldrich Poland (Poznań, Poland). The Pierce ${ }^{\mathrm{TM}}$ BCA Protein Assay Kit was from Thermo Fisher Scientific Inc. (Waltham, USA). All the chemicals used throughout the study were of analytical-reagent grade except for the derivatization reagent 2-chloro1-methylquinolinium tetrafluoroborate (CMQT), which was synthesized in our laboratory as described in a previous publication. ${ }^{12}$

\section{Subjects}

All the experiments were done in agreement with the Declaration of Helsinki (2008) of the World Medical Association. The study was approved by the Local Ethics Committee of the Medical University of Lodz (Poland). All the participants were informed of the terms of their involvement in the study and gave their informed consent before the study.

The group of 59 CAD-affected patients were recruited after the verification of the occurrence of coronary artery disease on the basis of echocardiography and/or angiography. Those with at least $50 \%$ occlusion in at least 1 coronary vessel were considered to be suffering from coronary artery disease. Fifty-four non-CAD control subjects were recruited from individuals whose echocardiography and/or angiography results showed that none of their coronary arteries were $50 \%$ occluded.

The exclusion criteria were similar to those used in earlier studies, with some modifications. ${ }^{13,14}$ Individuals who suffered from unstable angina pectoris, chronic renal failure, type 1 diabetes mellitus, allergic diseases, autoimmune diseases, infectious diseases, acute infection in the previous 2 weeks, acute coronary syndrome within the previous 6 months, those with any kind of inflammatory disease or non-chronic inflammation, malignancies, alcohol abusers, those who used alcohol the day before the experiment, drug abusers, those suffering from psychiatric disorders or any chronic diseases other than CAD, and those on specific diets or taking diet supplements were excluded. Patients whose clinical status was difficult to diagnose and pregnant women were also excluded.

The following data were recorded for each participant: age, gender, height, weight, hip and waist dimensions, smoking (past or current), exposure to stress, kind of professional activity (physical, mental, mixed, none), frequency of physical activity (high, moderate, none), the occurrence of myocardial infarction in the past (with the number of infarctions), ischemic stroke or transient ischemic attack (with the number of cerebrovascular events), the occurrence of cardiovascular procedures such as percutaneous coronary intervention (PCI) and/or coronary bypass grafting (CABG), other concomitant diseases and current pharmacotherapies (antihypertensive, hypolipidemic and antidiabetic drugs).

\section{Measurements of blood morphology and biochemistry}

Samples of peripheral blood were taken (always between 8:00 and 10:00 am) from a forearm vein, following 
at least $12 \mathrm{~h}$ of overnight fasting. For anticoagulation $0.105 \mathrm{M}$ buffered citrate was used in the case of plasma samples used for the analysis of thiol concentrations, while EDTA was used in the case of samples for the morphology analysis. Glucose was assessed in serum samples. To obtain plasma, the blood samples were centrifuged $\left(2000 \times \mathrm{g} / 15 \mathrm{~min} / 4^{\circ} \mathrm{C}\right)$ immediately after blood withdrawal. The plasma was portioned and the aliquots were stored at $-70^{\circ} \mathrm{C}$ until further use, without any thawing before testing. To obtain blood serum, the blood samples were collected in tubes with a clotting activator, and were left undisturbed at room temperature for $30 \mathrm{~min}$, then centrifuged $\left(2000 \times \mathrm{g} / 15 \mathrm{~min} / 4^{\circ} \mathrm{C}\right)$ and stored at $-70^{\circ} \mathrm{C}$ until further use, without any thawing before measurements.

Blood morphology and biochemical serum/plasma parameters were measured using the 5-Diff Sysmex XS-100i (Sysmex, Kobe, Japan) and the DIRUI CS 400 analyzer (Dirui, Changchun, China), respectively.

\section{HPLC measurements of low-molecular- weight thiol concentrations}

A 2-fold dilution of plasma aliquots with phosphate buffer $(\mathrm{pH}=7.6 ; 0.2 \mathrm{~mol} / \mathrm{L})$ was followed by sample supplementation with TCEP phosphate buffer solution $(0.25 \mathrm{~mol} / \mathrm{L})$. The mixture obtained was vortexed and put aside for $10 \mathrm{~min}$ and then supplemented with CMQT $(0.1 \mathrm{~mol} / \mathrm{L})$. After 2 more min, $3 \mathrm{~mol} / \mathrm{L}$ PCA was added to precipitate plasma proteins, which were further spun down $(10 \mathrm{~min}, 12000 \times \mathrm{g})$. The supernatant obtained was transferred into a vial, and a $20 \mu \mathrm{L}$ aliquot was injected into the ZORBAX SB C18 column $(150 \times 4.6 \mathrm{~mm})$ packed with $5 \mu \mathrm{m}$ particles.

The composition of the mobile phase was as follows: $0.1 \mathrm{~mol} / \mathrm{L}$ TCA (solution A), adjusted to $\mathrm{pH}=1.65$ with $1 \mathrm{~mol} / \mathrm{L} \mathrm{NaOH}$ solution, and acetonitrile (solution B); flow rate $1 \mathrm{~mL} / \mathrm{min}$; temperature $25^{\circ} \mathrm{C}$.

The elution profile was as follows: $0-8 \mathrm{~min}, 11-40 \% \mathrm{~B}$; 8-12 min, 40-11\% B; (A/B, v/v). Detection and quantification were conducted by UV absorbance at $355 \mathrm{~nm}$. Identification of the peaks was based on comparisons of retention times and diode-array spectra, taken at the real time of analysis, with a corresponding set of data obtained by analyzing the authentic compounds.

\section{Measurements of concentrations of free sulfhydryl groups and carbonyl groups in blood plasma proteins}

The concentrations of free sulfhydryl groups in plasma proteins were assessed according to the method reported in a previous publication, originally developed by Ando and Steiner. ${ }^{13,15}$ The concentration of carbonyl groups in plasma proteins was measured with the use of a spectrophotometric assay, according to the protocol report- ed in papers by Levine et al. and Rice-Evans et al. ${ }^{16,17}$ The concentration of plasma protein was assessed with the Pierce ${ }^{\mathrm{TM}} \mathrm{BCA}$ Protein Assay Kit, in accordance with the manufacturer's instructions.

\section{Statistical analysis}

Outliers, normal data distribution and homogeneity of variance were verified with Grubbs' test, the ShapiroWilk test and Levene's tests, respectively. Depending on the departures from normal distributions, data was presented as mean $\pm \mathrm{SD}$, or median and interquartile range (IQR: lower quartile, LQ [25\%], to upper quartile, UQ [75\%]). Some variables were transformed using the Box-Cox method. The unpaired Student's t-test on transformed data or the Mann-Whitney U test were employed to evaluate the significance of differences between the groups of non-CAD and CAD individuals. Multiple regression or Pearson's correlation (on raw data or the Box-Cox-transformed data) and an analysis of covariance (ANCOVA) were used to estimate the associations between the variables. All the statistical calculations were performed using STATISTICA.PL, v. 12.5 (StatSoft Polska, Kraków, Poland) and StatsDirect, v. 2.7.7 (StatsDirect Ltd, Cheshire, UK).

\section{Results}

\section{Basic characteristics of the study groups: Demography, anthropometry, socioeconomics, peripheral blood morphology and plasma biochemistry}

The patients affected by CAD had higher counts of total white blood cells (WBC) than the controls (Table 1). The counts of subpopulations of lymphocytes, neutrophils and monocytes were significantly increased in the CAD patients compared to the controls (data not shown).

Plasma creatinine and C-reactive protein (CRP) concentrations were increased in the patients suffering from CAD; these differences, however, did not reach statistical significance. Significantly higher fasting glucose was recorded in the CAD patients, and the difference remained significant when patients with diabetes were excluded from the CAD and non-CAD groups. Increased levels of triglycerides were noted in the CAD group. Total cholesterol and its lipoprotein subfractions were significantly lower in the CAD group than in the non-CAD controls. A comparison of low-molecular-weight thiols revealed significantly higher plasma homocysteine concentrations in the CAD-affected patients ( $p<0.005$ in the MannWhitney U test). The concentrations of cysteine, cysteinylglycine and glutathione were not significantly different between the non-CAD control group and the CAD subjects (Table 1). 

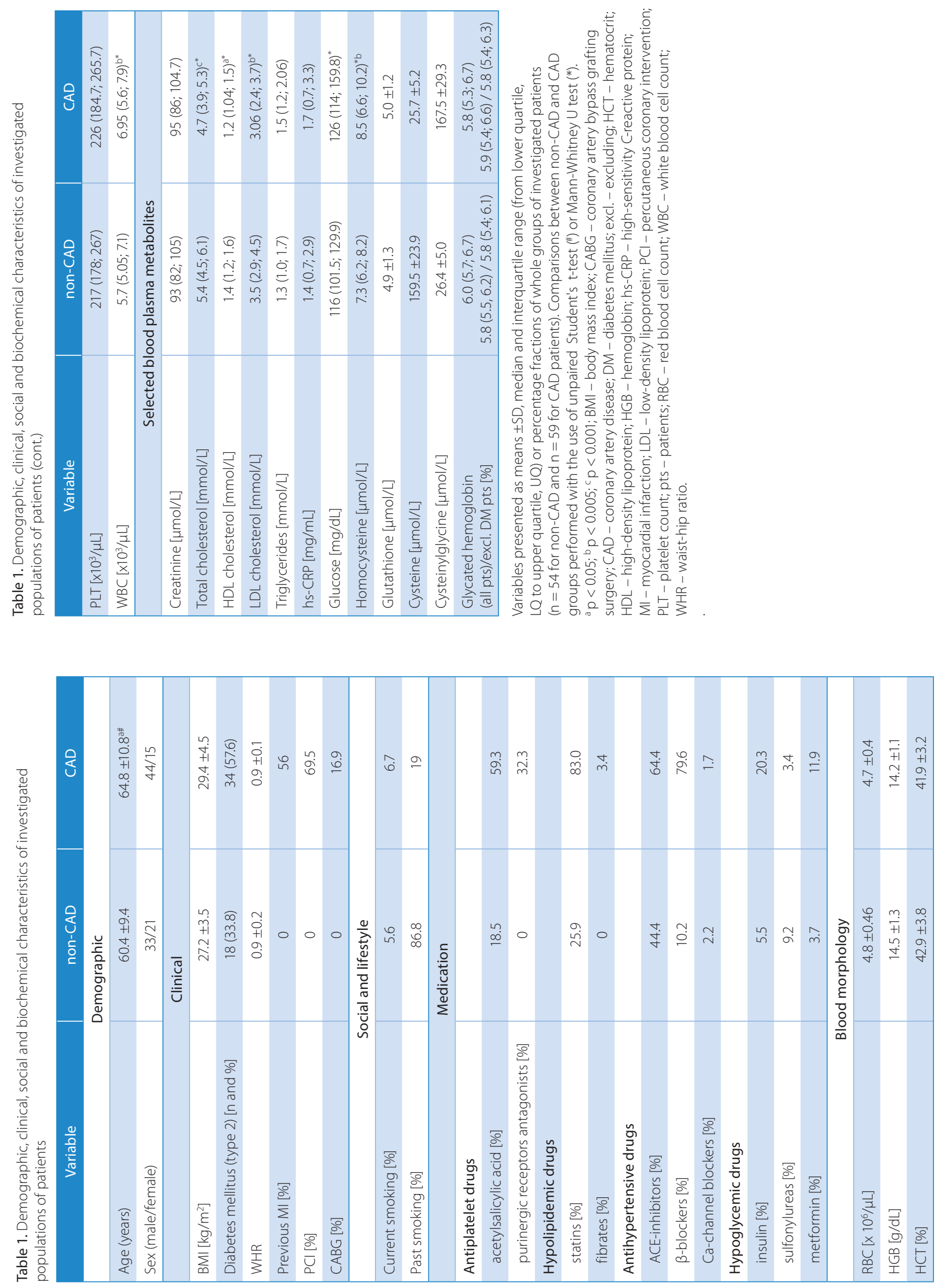


\section{Associations between plasma GSH levels, fasting glycemia and the occurrence of type 2 diabetes mellitus in the non-CAD and CAD patients}

When the patients in the CAD group were divided into 2 subgroups according to their plasma GSH levels - patients with plasma GSH lower than $\mathrm{Me}_{\mathrm{GSH}}=5.12 \mu \mathrm{mol} / \mathrm{L}$ (GSH group 1) and patients with plasma GSH higher or equal to the median value (GSH group 2), the patients with lower GSH demonstrated average fasting glycemia higher (by 13\%) than the patients with higher plasma GSH levels ( $p=0.037$ in the unpaired Student's t-test on the Box-Cox-transformed data) (Fig. 1A).

A similar analysis in the non-CAD control subjects, aimed at comparing fasting glucose levels in relation to GSH plasma concentrations, revealed no significant differences between the fasting glucose values of the patients with low GSH (plasma concentrations lower than $\mathrm{Me}_{\mathrm{GSH}}=4.84 \mathrm{umol} / \mathrm{L}$ ) and those with plasma GSH levels higher or equal to the median value (glucose concentrations of 118 [100-134] mg/dL vs 110 [103-126] mg/dL; $\mathrm{p}=0.434$ in the unpaired Student's t-test on the Box-Coxtransformed data) (Fig. 1A).

An analogical comparison between the control participants and the CAD-affected patients, with each group subdivided into 2 subpopulations according to the median values of blood glycemia (114 and $126 \mathrm{mg} / \mathrm{dL}$ in nonCAD and CAD subjects, respectively), revealed that the mean plasma GSH concentrations were approximately equal (ca. $5 \mu \mathrm{mol} / \mathrm{L}$ ) in both subpopulations of the nonCAD controls stratified according to median glycemia, but they were lower in the subpopulation of CAD patients with higher glycemia $(\geq 126 \mathrm{mg} / \mathrm{dL}): 4.8 \mu \mathrm{mol} / \mathrm{L}$ vs $5.3 \mu \mathrm{mol} / \mathrm{L} \mathrm{GSH}$; $\mathrm{p}=0.04$ in the non-paired, one-sided Student's t-test).

Plasma GSH and fasting glycemia were adjusted for age and the occurrence of diabetes mellitus, 2 variables that differed significantly between the non-CAD and CAD individuals (Fig. 1B). The adjusted values of plasma GSH
A

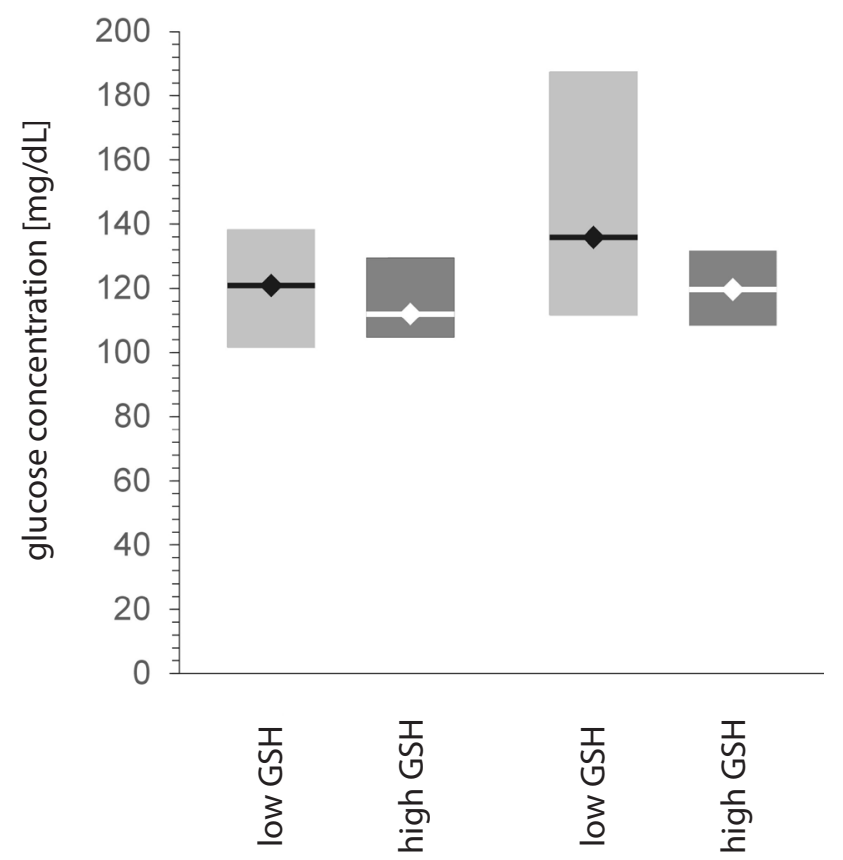

non-CAD patients

CAD patients
B

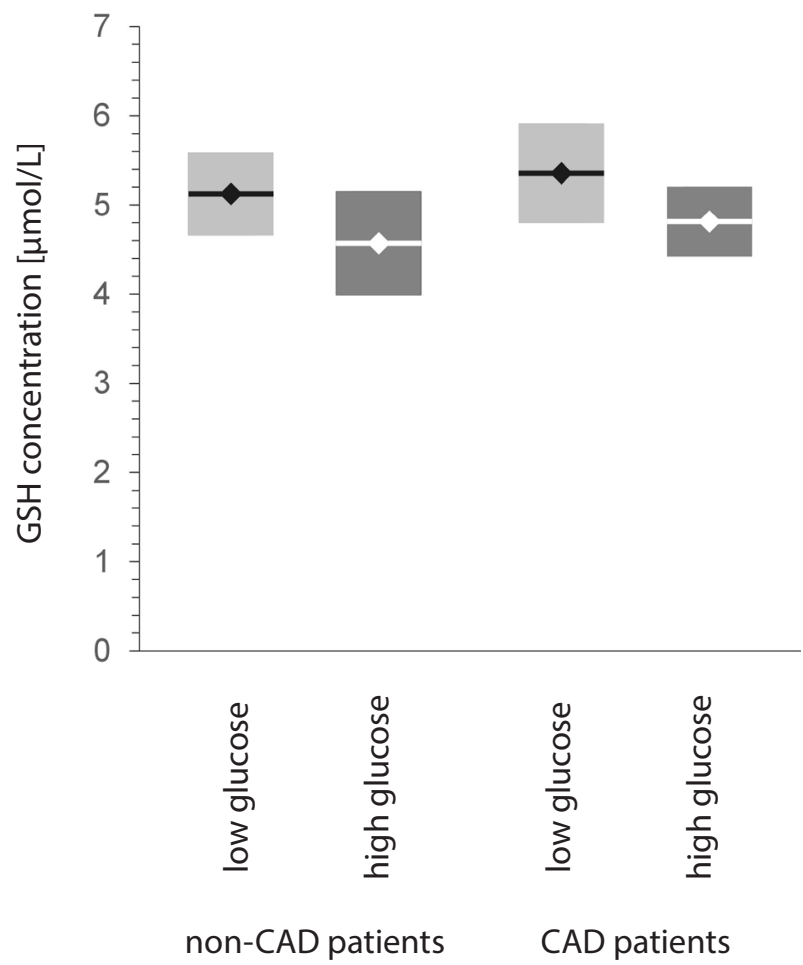

Fig.1. Glucose and reduced glutathione (GSH) concentrations in stratified groups of non-CAD controls and CAD patients

Data is presented as median and interquartile range for glucose (A) or mean $\pm 95 \% \mathrm{Cl}$ for GSH (B) in the subgroups of non-CAD controls and CAD subjects, stratified according to plasma GSH and fasting glycemia, respectively. For glucose, non-CAD controls and CAD patients were stratified into "Iow GSH" subgroups ( $n=27$ and $n=29$, respectively) with GSH levels lower than the group median ( Me $_{G S H}=4.807 \mu \mathrm{mol} / \mathrm{L}$ for the controls and $\mathrm{Me}_{\mathrm{GSH}}=5.154 \mu \mathrm{mol} / \mathrm{L}$ for the CAD patients), and "high GSH" subgroups ( $n=27$ and $n=30$, respectively) with GSH levels higher or equal to the established GSH median value. For GSH, non-CAD controls and CAD patients were stratified into "low glucose" subgroups ( $n=27$ and $n=29$, respectively) with fasting glycemia lower than the group median (Me Glucose $=117.1 \mathrm{mg} / \mathrm{dL}$ for the controls and $122.8 \mathrm{mg} / \mathrm{dL}$ for the CAD patients), and "high glucose" subgroups $(n=27$ and $n=30$, respectively) with fasting glycemia higher or equal to the established median value. Both GSH and glucose concentrations were adjusted for age and the occurrence of diabetes with the use of ANCOVA. The significance of changes was estimated with 2-way ANOVA and the post-hoc Fisher's LSD test. For glucose: the effect of CAD, $p<0.01$; the effect of GSH stratification, $p<0.05$; CAD, low GSH $>C A D$, high GSH, $p<0.02$. For GSH: the effect of CAD, $p=0.325$; the effect of glucose stratification, $p<0.03$. 
and fasting glycemia demonstrated a significant association in the cohort of all the study participants (both nonCAD and CAD) $(\mathrm{r}=-0.232 ; \mathrm{p}=0.014)$. No significant association between plasma GSH and fasting glycemia was found in the non-CAD controls $(r=-0.190 ; p=0.169)$. In the group of CAD-affected subjects, plasma GSH concentrations and glycemia were significantly negatively correlated ( $\mathrm{r}=-0.328 ; \mathrm{p}=0.011$ ) (Fig. $2 \mathrm{~A})$. In the CAD-affected patients, but not in the control subjects, the occurrence of type 2 diabetes mellitus significantly discriminated plasma GSH levels $(r=-0.125 ; \mathrm{p}=0.350$, between GSH and glucose adjusted for the occurrence of diabetes) (Fig. 2).

However, further subdivision of CAD- and DM-affected patients into subgroups according to the degree of impairment of glucose metabolism - normal glycemia (<88 mg/dL), higher normal glycemia (89-99 mg/dL), impaired fasting glycemia (100-125 mg/dL), diabetes mellitus $(>126 \mathrm{mg} / \mathrm{dL})$ - revealed a significant association between glycemia and plasma GSH levels only in the DM group $\left(r_{P}=-0.297 ; p<0.05\right)$, pointing to the significant role of DM in the occurrence of a GSH-glucose relationship. Further support for this hypothesis may be derived from the correlation calculus with and without standardization for CAD and DM. The overall association

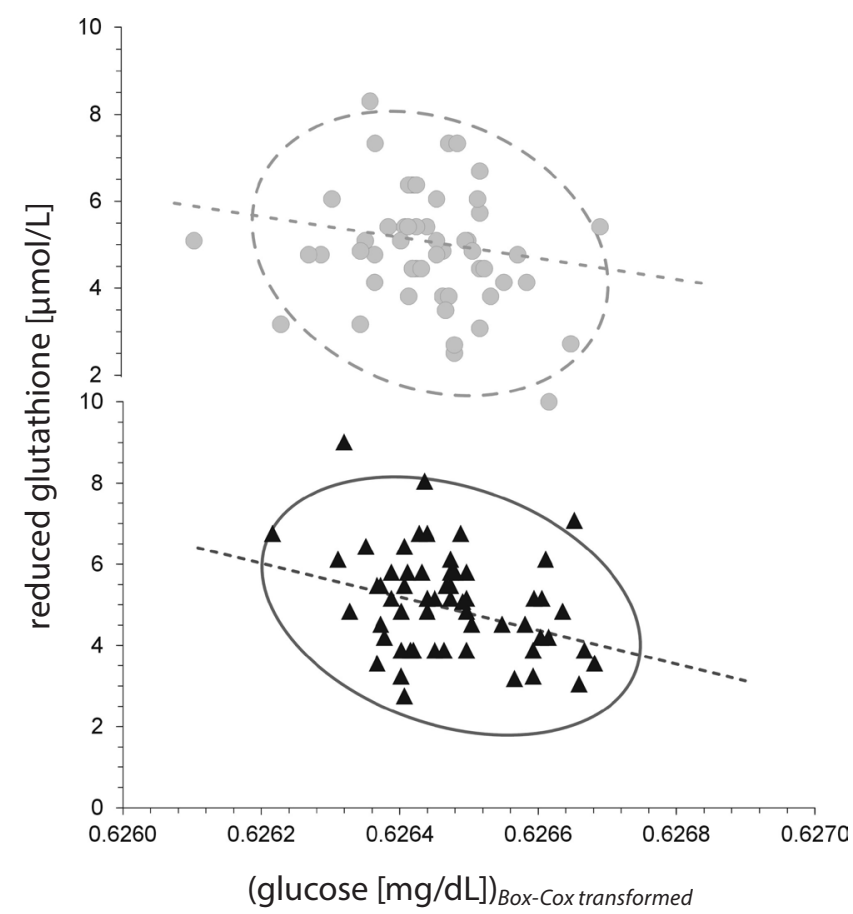

Fig. 2. Associations between plasma reduced glutathione (GSH) and fasting glycemia in non-CAD controls and CAD patients

Correlations between the concentration of GSH and fasting glycemia in non-CAD controls (grey circles) and CAD patients (black triangles), assessed by Pearson's linear correlation on Box-Cox transformed data. Both GSH and glucose concentrations were adjusted for age and the occurrence of diabetes with the use of ANCOVA. Scatter ellipses were estimated with the use of $95 \% \mathrm{Cl}$. Regression equations were: 1630.92595.9x for non-CAD ( $r=-0.190 ; p=0.169)$ and 2554.3-4069.3x for CAD patients $(r=-0.328 ; p=0.011)$; for both pooled groups: 1842.6-2933.4x for CAD patients $(r=-0.232 ; p=0.014)$. between GSH and glucose, without standardization for CAD or DM, was $\mathrm{r}_{\mathrm{P}}=-0.236(\mathrm{p}<0.02)$. When another variable was added to the model (CAD or DM), it appeared that only the first resulted in the maintenance of this significant association: $r_{\text {Psemipartial }}=-0.265(p<0.005)$ for the GSH-glucose correlation standardized for CAD and $\mathrm{r}_{\text {Psemipartial }}=-0.119(\mathrm{p}=0.199)$ for the GSH-glucose correlation standardized for DM. This clearly indicates that in both the CAD and non-CAD participants, the association between GSH and glucose is maintained, while DM reorients the extent of this association: it is maintained for DM patients, while it disappears for non-DM patients.

\section{Concentrations of sulfhydryl and carbonyl groups in blood plasma proteins and their association with levels of low-molecular- weight thiols in non-CAD and CAD patients}

To evaluate the pro-oxidant status of the peripheral blood plasma of the recruited subjects, the levels of carbonyl groups and free protein sulfhydryl groups in blood plasma proteins were measured. The concentrations of plasma protein carbonyls were not significantly different between the non-CAD and CAD patients: $0.10(0.08-0.14) \mu \mathrm{mol} / \mathrm{mg}$ of plasma protein in non-CAD subjects compared to 0.11 $(0.08-0.19) \mu \mathrm{mol} / \mathrm{mg}$ in CAD-affected subjects $(\mathrm{p}>0.05$ in the Mann-Whitney U test). Likewise, the levels of free sulfhydryl groups in plasma proteins were not different between non-CAD and CAD individuals (28.07 [19.51-41.17] $\mu \mathrm{mol} / \mathrm{mg}$ of plasma protein vs 27.72 [22.50-45] $\mu \mathrm{mol} / \mathrm{mg}$ in non-CAD and CAD-affected patients, respectively; p > 0.05 in the Mann-Whitney U test).

Neither marker of oxidative damage to blood plasma proteins was significantly associated with plasma levels of any of the low-molecular-weight thiols tested (GSH, Hcy, Cys or CysGly - data not shown) nor with glycemia, regardless of the inclusion or exclusion of DM-affected subjects.

\section{Discussion}

GSH seems to play some role in controlling plasma glucose levels and it reduces the progression of some hyperglycemia-associated cardiovascular complications, such as hypertension. ${ }^{18}$ It is possible that the development of cardiovascular complications might be facilitated in patients with higher glycemia and lower GSH concentrations, and these complications might therefore be more probable than in individuals with higher GSH.

The inverse relationship between plasma GSH and glucose concentrations in CAD reported in the present study is in agreement with results showing that hyperglycemia induces oxidative stress due to reductions of the pool of antioxidants like vitamin E, uric acid and vitamin C. ${ }^{19,20}$ On the basis of the present results, as well as those shown 
by others, it can be suggested that - very much alike $\alpha$-tocopherol, ascorbic acid and uric acid - GSH is very likely to be decreased by higher concentrations of glucose, as demonstrated in the CAD subjects in the current study, especially those with concomitant T2DM. ${ }^{21}$ Tessier et al. noted that the GSH/GSSG (glutathione disulfide) ratio is significantly lower in diabetic patients compared to healthy controls, either at baseline or after a glucose challenge. ${ }^{20}$ This clearly supports the current authors' assumption that the inverse association between glycemia and plasma GSH levels derives from the negative influence of high glucose on GSH levels. Hence, it can be confirmed that increased glucose concentrations are associated with decreased plasma GSH levels, and that this association appears to be independent of the concomitance of CAD. This conclusion, however, should be treated with caution, since the subpopulations of diabetic patients constituted only part of the non-CAD and CAD individuals in the present study (33.8\% of the controls and $57.6 \%$ of the CAD subjects).

The study shows that the stratification of the CAD patients according to plasma GSH levels significantly differentiates this group into those with lower and higher glucose. On the other hand, however, the inverse operation, i.e., the stratification according to fasting glucose levels, also discriminates the CAD patients into those with significantly lower and those with significantly higher plasma GSH concentrations. Thus, plasma GSH levels and fasting glycemia may simply be considered a 2 -way interplay: high glucose decreases the concentrations of GSH, while GSH reduces fasting glycemia. The authors therefore suggest, probably for the first time, that, contrary to previous reports, it is also probable that GSH may be the key factor determining glucose metabolism, and not only that glucose is a factor contributing to a diminished GSH pool. This conclusion may be supported by results demonstrating that an increased GSH/GSSG ratio, like that induced by vitamin $\mathrm{E}$ supplementation, positively associates with whole-body glucose disposal (WBGD) in hypertensive patients. ${ }^{22}$

This finding also implies that the link between GSH and glycemia may be not 1-directional but 2-way: high plasma glucose depletes the plasma GSH concentration, but an increased GSH concentration also improves glucose metabolism. Since GSH is a potent antioxidant, it seems reasonable to expect that lowering the GSH concentration should be connected with the presence of abundant markers of oxidative damage to biomacromolecules. Nevertheless, no significant increase in the levels of plasma protein carbonyls or decreased plasma protein free sulfhydryls was found in subjects with reduced concentrations of GSH and higher glycemia. This suggests that oxidative stress should not be considered the main mediator of the impact of glycemia on GSH and vice versa.

The present results should be considered with caution, since the papers cited present results from samples originating only from diabetic patients and mainly concern GSH concentrations inside erythrocytes rather than in blood plasma, whereas the results of the current study are all from samples of peripheral blood plasma in subgroups including some patients with diabetes mellitus. The authors can suggest that the distinct associations between blood glucose and GSH are less evident in subjects with apparently undisturbed (nondiabetic) glucose metabolism.

Thus, observed correlations between fasting glycemia and plasma GSH levels found in CAD-affected patients are strongly associated with coexistence of T2DM.

The inverse relationships between blood GSH and glucose offer some potentially interesting therapeutic clues. Strict control of GSH, or more broadly a proper balance of all low-weight thiols in human tissue fluids, may potentially significantly decrease the progression of CAD through the reduction of glucose levels. One promising compound in this regard seems to be $\mathrm{N}$-acetylcysteine, a known precursor of GSH that has been used successfully in the treatment of a few diseases, including vascular disturbances..$^{23-25}$

The results presented in this paper should be treated with caution for at least 2 reasons. Firstly the study involved quite few control and CAD-affected subjects, so the results certainly need to be reevaluated with a higher number of patients. Secondly as it can be seen in Table 1, the patients suffering from CAD were taking different drugs, often simultaneously. Thus, it cannot be excluded that the observed relationship between glucose and GSH should not be ascribed only to CAD and DM, but may also be at least partly a result of exposure to certain drugs and their metabolites. This issue should be also clarified in further studies.

\section{References}

1. Quadros AS, Sarmento-Leite R, Bertoluci M, et al. Angiographic coronary artery disease is associated with progressively higher levels of fasting plasma glucose. Diabetes Res Clin Pract. 2007;75:207-213.

2. Bittencourt C, Piveta VM, Oliveira CS, et al. Association of classical risk factors and coronary artery disease in type 2 diabetic patients submitted to coronary angiography. Diabetol Metab Syndr. 2014;6:46-54.

3. Gui MH, Qin GY, Ning G, et al. The comparison of coronary angiographic profiles between diabetic and nondiabetic patients with coronary artery disease in a Chinese population. Diabetes Res Clin Pract. 2009;85:213-219.

4. Su G, Mi S, Tao H, et al. Association of glycemic variability and the presence and severity of coronary artery disease in patients with type 2 diabetes. Cardiovasc Diabetol. 2011;10:19-28.

5. Aronson D. Hyperglycemia and the pathobiology of diabetic complications. Adv Cardiol. 2008;45:1-16.

6. Trevisan M, Browne R, Ram M, et al. Correlates of markers of oxidative status in the general population. Am J Epidemiol. 2001;154:348-356.

7. Ciuchi E, Odetti P, Prando R. Relationship between glutathione and sorbitol concentrations in erythrocytes from diabetic patients. Metabolism. 1996;45:611-613.

8. Cavalca V, Veglia F, Squellerio I, et al. Glutathione, vitamin E and oxidative stress in coronary artery disease: Relevance of age and gender. Eur J Clin Invest. 2009;39:267-272.

9. Damy T, Kirsch M, Khouzami L, et al. Glutathione deficiency in cardiac patients is related to the functional status and structural cardiac abnormalities. PLoS One. 2009;4:e4871-4878. 
10. Pietruszyński R, Markuszewski L, MasiarekK, Makowski M, Retelewska W, Watala C. Role of preprocedural glutathione concentrations in the prediction of major adverse cardiac events in patients with acute coronary syndrome treated with percutaneous coronary intervention. Pol Arch Med Wewn. 2013;123:228-237.

11. Powell LA, Warpeha KM, Xu W, Walker B, Trimble ER. High glucose decreases intracellular glutathione concentrations and upregulates inducible nitric oxide synthase gene expression in intestinal epithelial cells. J Mol Endocrinol. 2004;33:797-803.

12. Bald E, Głowacki R. 2-Chloro-1-methylquinolinium tetrafluoroborate as an effective and thiol specific UV-tagging reagent for liquid chromatography. J Liq Chromatogr Related Technol. 2001;24:1323-1339.

13. Karolczak K, Kamysz W, Karafova A, Drzewoski J, Watala C. Homocysteine is a novel risk factor for suboptimal response of blood platelets to acetylsalicylic acid in coronary artery disease: A randomized multicenter study. Pharmacol Res. 2013;74:7-22.

14. Karolczak K, Pietruszyński R, Drzewoski J, Kasznicki J, Watala C. Aspirin dose increase from 75 to $150 \mathrm{mg}$ suppresses red blood cell contribution to suboptimal platelet response to aspirin in patients with CAD. Cardiovasc Drugs Ther. 2013;27:549-558.

15. Ando Y, Steiner M. Sulfhydryl and disulfide groups of platelet membranes. I. Determination of sulfhydryl groups. Biochim Biophys Acta. 1973;311:26-37.

16. Levine RL, Garland D, Oliver CN, et al. Determination of carbonyl content in oxidatively modified proteins. Methods Enzymol. 1990;186:464-478.

17. Rice-Evans CA, Diplock AT, Seymons MCR. Techniques in Free Radical Research. Amsterdam, London, New York (NY), Tokyo: Elsevier; 1991.

18. Cerielo A, Motz E, Cavarape A, et al. Hyperglycemia counterbalances the antihypertensive effect of glutathione in diabetic patients: Evidence linking hypertension and glycemia through the oxidative stress in diabetes mellitus. J Diabetes Complications. 1997;11:250-255.

19. Ceriello A, Bortolotti N, Crescentini A, et al. Antioxidant defences are reduced during the oral glucose tolerance test in normal and non-insulin-dependent diabetic subjects. Eur J Clin Invest. 1998;28:329-333.

20. Tessier D, Khalil A, Fülöp T. Effects of an oral glucose challenge on free radicals/antioxidants balance in an older population with type II diabetes. J Gerontol A Biol Sci Med Sci. 1999;54:M541-545.

21. Konukoğlu D, Hatemi H, Ozer EM, Gönen S, Akçay T. The erythrocyte glutathione levels during oral glucose tolerance test. J Endocrinol Invest. 1997;20:471-475.

22. Barbagallo M, Dominguez LJ, Tagliamonte MR, Resnick LM, Paolisso $\mathrm{G}$. Effects of vitamin $\mathrm{E}$ and glutathione on glucose metabolism: Role of magnesium. Hypertension. 1999;34:1002-1006.

23. Arranz L, Fernández C, Rodríguez A, Ribera JM, De la Fuente M The glutathione precursor $\mathrm{N}$-acetylcysteine improves immune function in postmenopausal women. Free Radic Biol Med. 2008;45:1252-1262.

24. Lavoie S, Murray MM, Deppen $\mathrm{P}$, et al. Glutathione precursor, $\mathrm{N}$-acetyl-cysteine, improves mismatch negativity in schizophrenia patients. Neuropsychopharmacology. 2008;33:2187-2199.

25. Andrews NP, Prasad A, Quyyumi AA. N-acetylcysteine improves coronary and peripheral vascular function. J Am Coll Cardiol. 2001;37:117-123. 